\title{
Clinicopathological characteristics, treatment, and survival outcomes of cystadenocarcinoma of the salivary gland: a population-based study
}

\author{
Cheng-Fu Cai ${ }^{1,2, *}$ \\ Jia-Yuan Sun ${ }^{3, *}$ \\ Zhen-Yu $\mathrm{He}^{3}$ \\ Huan-Xin $\operatorname{Lin}^{3}$ \\ Fa-Rong Liu ${ }^{2,4}$ \\ Yan Wang ${ }^{3}$ \\ 'Department of Otolaryngology-Head \\ and Neck Surgery, Affiliated First \\ Hospital, Medical College, Xiamen \\ University, ${ }^{2}$ Teaching Hospital of \\ Fujian Medical University, Xiamen, \\ ${ }^{3}$ Department of Radiation Oncology, \\ State Key Laboratory of Oncology in \\ South China, Collaborative Innovation \\ Center of Cancer Medicine, Sun \\ Yat-Sen University Cancer Center, \\ Guangzhou, ${ }^{4}$ Department of \\ Psychology, Affiliated First Hospital, \\ Medical College, Xiamen University, \\ People's Republic of China \\ *These authors contributed equally \\ to this work
}

Correspondence: Fa-Rong Liu Department of Psychology, Affiliated First Hospital, Medical College, Xiamen University, 55 Zhenhai Road, Xiamen 361003, People's Republic of China

Tel +895922137552

$\mathrm{Fax}+895922137189$

Email liufr@fh.xmu.edu.cn

Yan Wang

Department of Radiation Oncology, State Key Laboratory of Oncology in South China, Collaborative Innovation Center of Cancer Medicine, Sun Yat-sen University Cancer Center, 65I Dongfeng Road East, Guangzhou 5I 0060, People's Republic of China

Tel +862087343543

Fax +862087343392

Email wangyan@sysucc.org.cn
This article was published in the following Dove Press journal:

OncoTargets and Therapy

25 October 2016

Number of times this article has been viewed

Background: The aim of this study was to investigate the clinicopathological characteristics, treatment, and survival of cystadenocarcinoma of the salivary gland.

Patients and methods: Cases in the Surveillance, Epidemiology, and End Results database from 1991 to 2012 were identified. Factors significantly associated with survival were identified using Kaplan-Meier survival analysis and Cox proportional hazard regression.

Results: A total of 65 patients were identified; of these patients, 64 received surgical treatment, 25 underwent lymphadenectomy, and four (16.0\%) patients had nodal metastasis and only one $(2.1 \%)$ patient had poorly differentiated disease. The most common tumor location was the parotid gland (87.7\%). The median follow-up was 55 months. None of the patients died of salivary gland malignant-tumor-related disease. The 5- and 10-year cause-specific survival rates were $97.0 \%$ and $81.4 \%$, respectively. The 5 - and 10 -year overall survival rates were $84.6 \%$ and $60.7 \%$, respectively. Surgical procedures, lymphadenectomy, and adjuvant radiotherapy did not affect survival.

Conclusion: Salivary gland cystadenocarcinoma is extremely rare but has an excellent prognosis, and surgery is the mainstay of treatment.

Keywords: cystadenocarcinoma, salivary gland, SEER, treatment, survival

\section{Introduction}

Cystadenocarcinoma of the salivary gland is an extremely rare malignant tumor that was first described by the World Health Organization in $1991 .^{1}$ The estimated incidence of cystadenocarcinoma is $\sim 2-5 \%$ of all salivary gland malignancies. ${ }^{1-3}$ Diagnosis is based on pathologic findings, in which there is a predominantly cystic growth pattern that often exhibits intraluminal papillary proliferation. ${ }^{4}$ Currently, there are few reported cases related to cystadenocarcinoma of the salivary gland in the literature, ${ }^{5-10}$ and the related clinicopathological characteristics, treatment, and outcome results are limited. ${ }^{3}$ In this study, we used a population-based national registry (Surveillance, Epidemiology, and End Results, SEER) to investigate the clinicopathological characteristics, treatment, and survival outcomes of patients with cystadenocarcinoma of the salivary gland.

\section{Patients and methods}

\section{Patients and clinicopathological characteristics}

Patients with a pathologic diagnosis of cystadenocarcinoma of the salivary gland were identified using the SEER database from 1991 to 2012. ${ }^{11}$ Pathologic diagnosis was based on the primary site using the International Classification of Disease for Oncology, 
Third Edition. This study was approved by the ethics committee of the Affiliated First Hospital, Medical College, Xiamen University, who did not require that informed consent be obtained, as the study used SEER database data.

The following clinicopathological characteristics were obtained from the SEER database: year of diagnosis, race, age, tumor location, grade, SEER stage, and nodal status. Local treatment modalities including surgery, lymphadenectomy, and radiotherapy were also identified. Survival status, including cause of death, and the duration of follow-up were recorded.

\section{Statistical analysis}

Univariate and multivariate Cox regression analyses were used to analyze the risk factors for cause-specific survival (CSS) and overall survival (OS). Calculation of survival rates was plotted using the Kaplan-Meier method and compared using the log-rank testing. All data were analyzed using the SPSS software package, version 21.0 (IBM Corporation, Armonk, NY, USA). A $P$-value of $<0.05$ was considered statistically significant.

\section{Results}

\section{Clinicopathological characteristics}

A total of 16,923 patients were diagnosed with salivary gland malignant tumors from 1991 to 2012, including 65 $(0.4 \%)$ patients with cystadenocarcinoma (Table 1$)$. The median age was 62.0 years (range, 10-95 years); 90.8\% of patients were diagnosed after 2000, and there was equal disease incidence in male and female patients. The most common tumor location was the parotid gland (57 patients, $87.7 \%$ ), followed by the submandibular gland (four patients, $6.2 \%)$; four patients $(6.2 \%)$ had no specific site records. Most of the patients $(98.5 \%)$ were at localized and regional SEER stage, and only one patient (1.5\%) was unstaged. Of the 47 patients with histological grade available, $42.6 \%$, $55.3 \%$, and $2.1 \%$ had Grade 1, Grade 2, and Grade 3 disease, respectively.

\section{Treatment}

Of the 65 patients, $64(98.5 \%)$ received surgical treatment. Of the 61 patients with surgical procedure information available, $43(70.5 \%)$ underwent local tumor excision or less than total parotidectomy, and 18 (29.5\%) underwent total parotidectomy or radical parotidectomy. A total of 25 patients underwent lymphadenectomy, and only four patients $(16.0 \%)$ had nodal metastasis. A total of 16 patients underwent adjuvant radiotherapy.
Table I Clinicopathological characteristics of patients $(n=65)$

\begin{tabular}{|c|c|}
\hline Characteristics & \\
\hline \multicolumn{2}{|l|}{ Age (years) } \\
\hline Median (range) & $62.0(10-95)$ \\
\hline$<50$ & 7 \\
\hline$\geq 50$ & 58 \\
\hline \multicolumn{2}{|l|}{ Year of study } \\
\hline $199 \mid-1995$ & 2 \\
\hline $1996-2000$ & 8 \\
\hline $200 I-2005$ & 15 \\
\hline $2006-2012$ & 40 \\
\hline \multicolumn{2}{|l|}{ Race } \\
\hline White & 52 \\
\hline Black & 5 \\
\hline Other & 8 \\
\hline \multicolumn{2}{|l|}{ Sex } \\
\hline Male & 34 \\
\hline Female & 31 \\
\hline \multicolumn{2}{|l|}{ Tumor location } \\
\hline Parotid gland & 57 \\
\hline Submandibular gland & 4 \\
\hline Others & 4 \\
\hline \multicolumn{2}{|l|}{ Grade $(n=47)$} \\
\hline I & 20 \\
\hline 2 & 26 \\
\hline 3 & I \\
\hline \multicolumn{2}{|l|}{ SEER stage } \\
\hline Localized & 56 \\
\hline Regional & 8 \\
\hline Unstaged & I \\
\hline \multicolumn{2}{|l|}{ Surgery } \\
\hline No & I \\
\hline Yes & 64 \\
\hline \multicolumn{2}{|l|}{ Surgical procedures $(n=6 I)$} \\
\hline Partial parotidectomy & 43 \\
\hline Total parotidectomy & 18 \\
\hline \multicolumn{2}{|l|}{ Radiotherapy } \\
\hline No & 49 \\
\hline Yes & 16 \\
\hline \multicolumn{2}{|l|}{ Lymphadenectomy $(n=64)$} \\
\hline No & 39 \\
\hline Yes & 25 \\
\hline \multicolumn{2}{|l|}{ Nodal status $(n=25)$} \\
\hline Negative & 21 \\
\hline Positive & 4 \\
\hline
\end{tabular}

Abbreviation: SEER, Surveillance, Epidemiology, and End Results.

\section{Outcomes}

The median follow-up was 55 months (range, 1-204 months). A total of 13 patients died; ten patients died of noncancer disease, and three patients died of other malignant tumors; none of the patients died of salivary gland malignant-tumorrelated disease. The 5- and 10-year CSS rates were 97.0\% and $81.4 \%$, respectively (Figure 1A). The 5- and 10-year OS rates were $84.6 \%$ and $60.7 \%$, respectively (Figure $1 \mathrm{~B}$ ). 


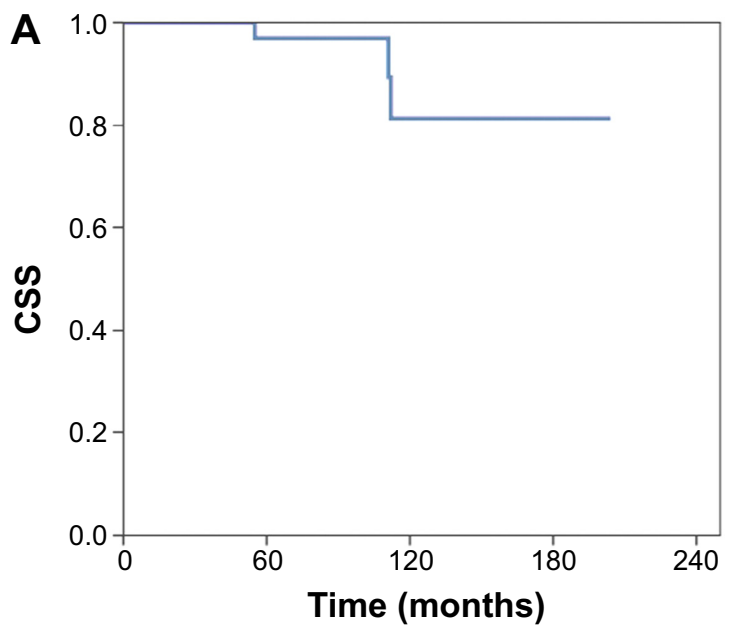

Figure I CSS (A) and OS (B) of cystadenocarcinoma of the salivary gland. Abbreviations: CSS, cause-specific survival; OS, overall survival.

\section{Influence of local treatment on survival}

The effects of local treatment for cystadenocarcinoma of the salivary gland on CSS and OS were further analyzed, and the results showed that surgical procedures, lymphadenectomy, and adjuvant radiotherapy did not affect the CSS and OS (all, $P>0.05$ ).

\section{Discussion}

Cystadenocarcinoma of the salivary gland had an excellent prognosis, but most studies currently have few patients. ${ }^{5-10}$ The study with the most patients in the literature was by Foss et al, ${ }^{3}$ which involved 57 patients; the median age was 58.8 years, and the main disease site was the parotid gland (61.4\%). In our study, we used the SEER database to assess the clinicopathological characteristics, treatment, and survival outcomes of cystadenocarcinoma of the salivary gland, and our findings are similar to those of Foss et al. ${ }^{3}$ Regarding cystadenocarcinoma of the salivary gland, a highgrade malignant tumor usually leads to a relatively high risk of disease recurrence with rapid progression. ${ }^{12,13}$ However, in our study, only one patient was pathologically diagnosed with poorly differentiated disease.

Currently, the main treatment for cystadenocarcinoma of the salivary gland is radical surgery. Lymphadenectomy is recommended for patients with lymph node enlargement. ${ }^{14}$ The main site of recurrence following radical resection is the cervical lymph nodes, and there is less recurrence in the primary tumor bed. ${ }^{3,15}$ Mills et al ${ }^{16}$ reported that three of five patients $(60 \%)$ with cystadenocarcinoma of the salivary gland had cervical lymph node metastases after 2-21 years. Similarly, Mukaigawa et $\mathrm{al}^{15}$ reported on four patients with cystadenocarcinoma of the salivary gland who underwent

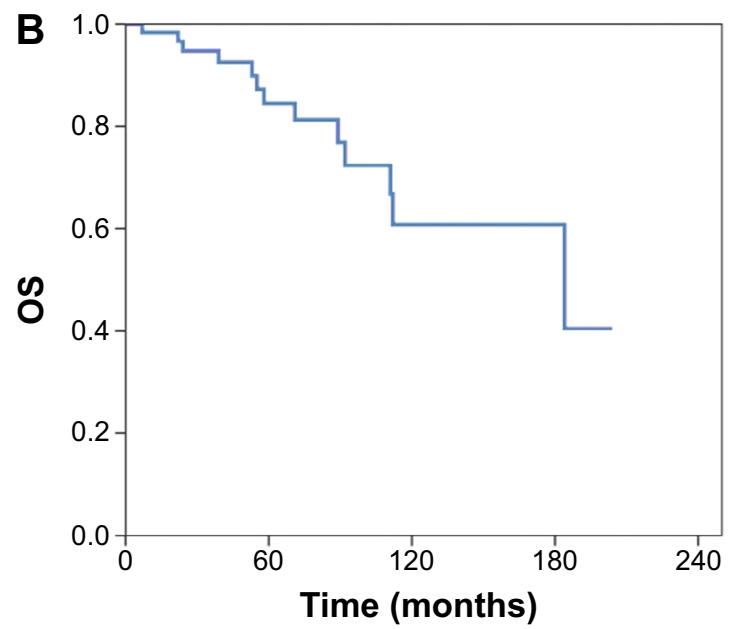

radical resection, where two patients underwent lymph node dissection, and three patients had cervical lymph node recurrence after 7,17 , and 24 months (with parotid lymph node recurrence after 43 months), respectively. Foss et al reported that one patient had regional lymph node metastasis after 55 months and three patients had lymphatic metastasis at initial diagnosis. ${ }^{3}$ In our study, 25 patients underwent lymphadenectomy, and $16.0 \%$ of patients were node positive. Due to the limitations of the SEER data, we could not clarify the lymph node status of the patients. However, based on the results of the abovementioned studies, the clinical course of follow-up for patients should also pay close attention to lymph node metastasis.

Radiotherapy is usually recommended for high-grade, recurrent disease, or for inoperable patients. ${ }^{14}$ Foss et $\mathrm{al}^{3}$ also recommended postoperative radiotherapy for patients with cervical lymph node metastasis. Our results also show that surgery is the main therapeutic method for cystadenocarcinoma of the salivary gland; other local treatment modalities such as lymphadenectomy and adjuvant radiotherapy had no effect on survival, which may be related to the excellent control rate of surgical resection.

In this study, none of the patients with cystadenocarcinoma of the salivary gland died of salivary gland malignant-tumor-related disease. Although mortality in cystadenocarcinoma of the salivary gland tends to be from other causes, long-term follow-up is essential, as some patients might relapse after a very long time, which may be associated with the slow growth and low-grade destructive features of cystadenocarcinoma of the salivary gland. ${ }^{16}$

The current study has several limitations that need to be considered. The main limitation is the inherent biases that 
exist in any retrospective study. Second, the SEER database does not record data such as systemic therapy regimen and dose, margin status, and local and regional recurrence. In addition, there is little information to guide the analysis of why lymphadenectomy and postoperative radiotherapy were or were not completed in certain patients. Furthermore, some adenocarcinomas recorded in the SEER database such as cystadenocarcinomas may be other entities that have been only recently recognized, ie, cribriform adenocarcinoma of tongue, mammary analog secretory carcinoma, and acinic cell carcinoma, which may be difficult to discriminate from cystadenocarcinoma and may require advanced techniques (immunohistochemistry and molecular pathology).

\section{Conclusion}

Cystadenocarcinoma is an extremely rare type of salivary gland malignant tumor with excellent prognosis, and surgery is the mainstay of treatment.

\section{Disclosure}

The authors report no conflicts of interest in this work.

\section{References}

1. Seifert G, Sobin LH, editors. Histological Typing of Salivary Gland Tumours. 2nd ed. Berlin: Springer-Verlag; 1991.

2. Seifert G, Sobin LH. The World Health Organization's histological classification of salivary gland tumors. A commentary on the second edition. Cancer. 1992;70(2):379-385.

3. Foss RD, Ellis GL, Auclair PL. Salivary gland cystadenocarcinomas. A clinicopathologic study of 57 cases. Am J Surg Pathol. 1996;20(12): 1440-1447.

4. Barnes L, Eveson JW, Reichart P, Sidransky D. World Health Organization classification of tumours. Pathology and Genetics of Head and Neck Tumours. Lyon: IARC Press; 2005:232.
5. Koç M, Yanilmaz M, Yildirim H, Gök U, Cobanoğlu B. MRI findings of papillary cystadenocarcinoma of the submandibular gland. Diagn Interv Radiol. 2010;16(1):20-23.

6. Gallego L, Junquera L, Fresno MF, de Vicente JC. Papillary cystadenoma and cystadenocarcinoma of salivary glands: two unusual entities. Med Oral Patol Oral Cir Bucal. 2008;13(7):E460-E463.

7. Harimaya A, Somekawa Y, Sasaki M, Ohuchi T. Cystadenocarcinoma (papillary cystadenocarcinoma) of the submandibular gland. J Laryngol Otol. 2006;120(12):1077-1080.

8. Nakagawa T, Hattori K, Iwata N, Tsujimura T. Papillary cystadenocarcinoma arising from minor salivary glands in the anterior portion of the tongue: a case report. Auris Nasus Larynx. 2002;29(1):87-90.

9. Enomoto K, Yamashita H, Harada H, et al. A case of cystadenocarcinoma of the ectopic salivary gland: comparison of pre-operative ultrasound, CT and MR images with the pathological specimen. Dentomaxillofac Radiol. 2012;41(4):349-354.

10. Mlika M, Chelly I, Azouz H, et al. Salivary gland cystadenocarcinoma of the tongue: a case report and literature review. Med Case Stud. 2010; 1(1):1-4.

11. Surveillance, Epidemiology, and End Results (SEER) Program (www. seer.cancer.gov) SEER*Stat Database: Incidence - SEER 18 Regs Research Data + Hurricane Katrina Impacted Louisiana Cases, Nov 2014 Sub (1973-2012 varying) - Linked To County Attributes - Total U.S., 1969-2013 Counties, National Cancer Institute, DCCPS, Surveillance Research Program, Surveillance Systems Branch, released April 2015, based on the November 2014 submission.

12. Pollett A, Perez-Ordonez B, Jordan RC, Davidson MJ. High-grade papillary cystadenocarcinoma of the tongue. Histopathology. 1997;31(2): 185-188.

13. Cavalcante RB, da Costa Miguel MC, Souza Carvalho AC, Maia Nogueira RL, Batista de Souza L. Papillary cystadenocarcinoma: report of a case of high-grade histopathologic malignancy. Auris Nasus Larynx. 2007;34(2):259-262.

14. Ellis GL, Auclair PL, Gnepp DR. Surgical Pathology of Salivary Glands. Philadelphia: WB Saunders; 1991:464-470.

15. Mukaigawa T, Hayashi R, Miyazaki M, Shinozaki T, Tomioka T, Fujii S. Cystadenocarcinoma of the salivary glands with potential lymph node metastasis. Auris Nasus Larynx. 2016;43(3):340-344.

16. Mills SE, Garland TA, Allen MS Jr. Low-grade papillary adenocarcinoma of palatal salivary gland origin. Am J Surg Pathol. 1984;8(5): 367-374.
OncoTargets and Therapy

\section{Publish your work in this journal}

OncoTargets and Therapy is an international, peer-reviewed, open access journal focusing on the pathological basis of all cancers, potential targets for therapy and treatment protocols employed to improve the management of cancer patients. The journal also focuses on the impact of management programs and new therapeutic agents and protocols on

\section{Dovepress}

patient perspectives such as quality of life, adherence and satisfaction. The manuscript management system is completely online and includes a very quick and fair peer-review system, which is all easy to use. Visit http://www.dovepress.com/testimonials.php to read real quotes from published authors. 